\title{
Detection of Supernumerary Tooth in the Nasal Cavity
}

\author{
Stuti Mathur* \\ Department of ENT, Patil Hospital, India
}

*Corresponding author: Stuti Mathur, Department of ENT, Patil Hospital, India

\begin{abstract}
Aims and objectives: To detect super numeracy tooth in nasal cavity.

Introduction: The ectopic dentition present in intranasal area is very rare finding clinically. It is very difficult to make proper diagnosis of such condition. Teeth is present in ovaries, testes and pre-sacral regions. The presence of teeth in maxillary sinus, mandibular condyle, chin, nose and orbit in maxillofacial region is also observed.
\end{abstract}

Case Report: Prospective cross-sectional study was conducted. Anterior microscopy was done; proper detection of supernumerary tooth was carried out.

Result: A 21-year-old female patient presented with complaints of intermittent right sided epistaxis. She had history of occasional headache for 6 months. An anterior rhinoscopic examination revealed a hard white-mass which is peripherally surrounded by granulation tissue that lies in floor of the right-nasal cavity. It's appearance clinically was of a rhinolith.

Conclusion: Super-numeracy teeth are observed extra to normal complement indentation. They may occur unilaterally or bilaterally, single or multiple. A variety of complications can occur that may be crowding or cyst formation. There by, identifying early and also treatment are useful for management.

Keywords: Supernumerary; ectopic; dentition; Epistaxis; Rhinoscopic examination

\section{Introduction}

Ectopic dentition situated in intranasal region is very rare thing which is clinically observed. The proper measures should be adapted to detect supernumerary tooth. Teeth are present in ovaries, anterior mediastinumandpresacral regions, testes [1,2], also found in maxillary- sinus, mandibular-condyle, coronoid-process, chin, nose and orbit in the maxillofacial region, while rarely seen in intranasal region. Most common conditions which are associated with a raised prevalence of ectopic teeth are cleft lip and palate, cleido-cranial dysplasia and Gardner syndrome. Intra-nasal teeth can lead to problems e.g. Nasal-obstruction, chronic-rhinorrhoea and speech-defects. Most common ectopic-tooth which arises in the maxillary midline is known as a mesiodens. This unusual condition should be supposed to be present in patients with nasal blockage/ obstruction and unilateral purulent rhinorrhoea [3-7]. So, this study was conducted to detect the supernumerary tooth in nasal cavity.

\section{Case Report}

Atwenty-one-year-old female patient presented with complaints of intermittent right sided epistaxis and history of occasional headache for 6 months. An anterior rhinoscopic examination revealed a hard- white mass surrounded by granulation tissue lying in the floor of the right nasal cavity. The clinical appearance was that of a rhinolith. An orthopantomography revealed the presence of a radio-opaque tooth shape mass, suggestive of a supernumerary tooth since the intraoral dental occlusion was complete. Computed Tomography (CT) scan was required to assess the exact position of the presumed-supernumerary tooth and its relationship with the surrounding structures. The CT examination showed that the mass lesion was like a conical shaped structure tapering to a point suggestive of a tooth lying in the anteroposterior direction (Figure 1). The patient had gone an endoscopic removal of the supernumerary tooth under general anesthesia. Once tooth was 
localised, the covering periosteum was removed, location of tooth was deviated from its area of impaction and it was removed with forceps from the right nostril (Figures 2 \& 3). Uneventful postoperative course was observed.

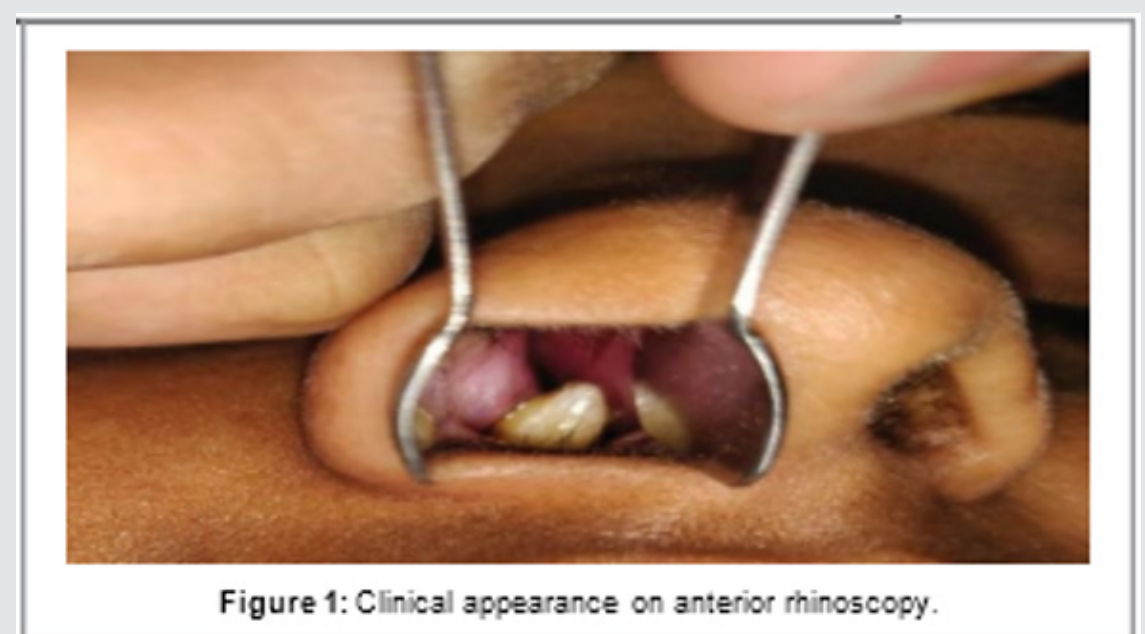

Figure 1: Clinical appearance on anterior rhinoscopy

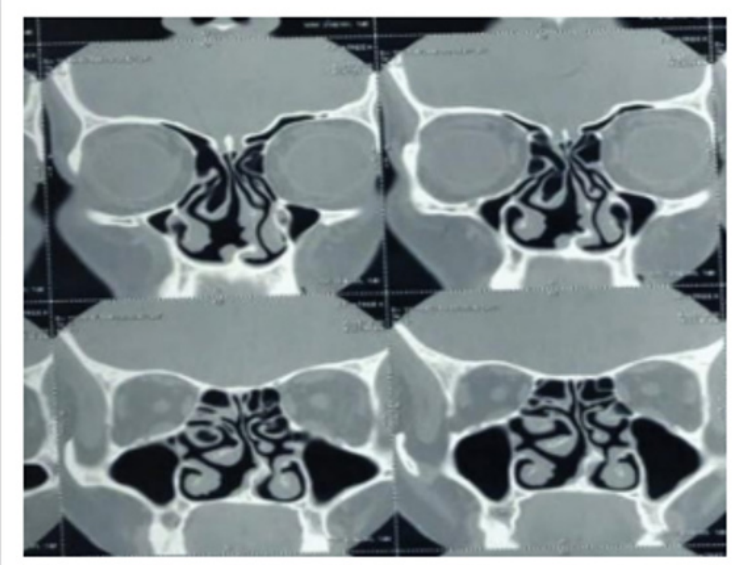

Figure 2: CT scan showing relation if tooth with surrounding structures.

Figure 2: CT scan showing relation if tooth with surrounding structures

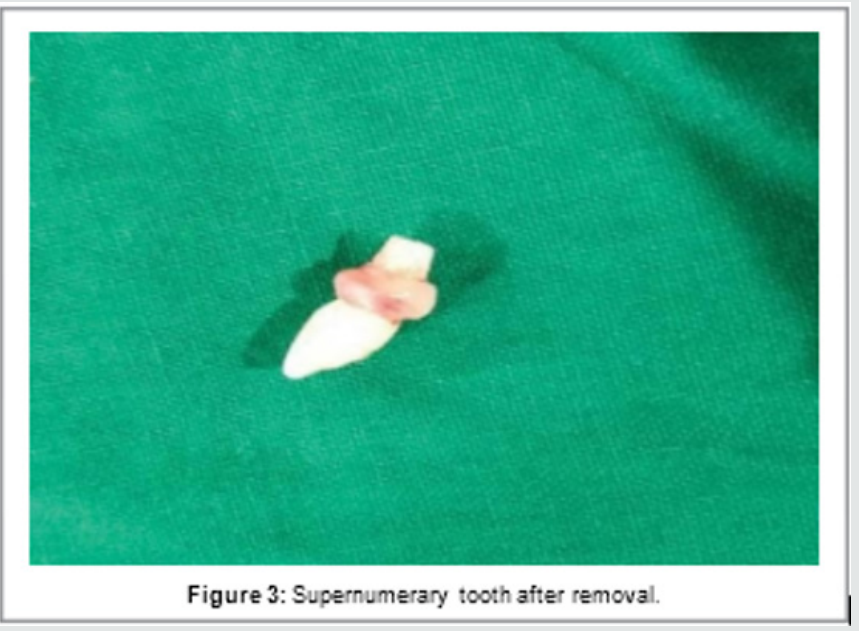

Figure 3: Supernumerary tooth after removal 


\section{Discussion}

It is observed that 0.1 to 1 percent of population is influenced by and having supernumerary tooth, with most common situation as upper incisor area called as mesiodens. The causes of supernumerary teeth are not totally understood. According to one theory, supernumerary tooth is generated either from a thin tooth bedwhicharisesfrom the dental lamina close to permanent tooth bud /or from division of the permanent bud itself $[1,2]$. While another theory states that their development is due to reversion of dentition of primates that are extinct and had 3 pairs of incisors. There is formation of supernumeraries due to local, independent, conditioned hyperactivity of dental lamina, as suggested by hyperactivity theory. There is a role of heredity also, because supernumeraries are commonly seen in relatives of children who are affected as compared to general population. But, simple Mendelian pattern is not followed by anomaly [3-5]. The reason of this ectopic growth formation in not clearly understood, it hasbeen attributed to obstructionatthe time of tooth eruption secondary to crowded dentition, persistent deciduous teeth/or exceptionally dense bone. Some pathogenic factors responsible are genetic predisposition, developmental disturbances, rhino genic or odontogenic infection and displacement as a result of trauma/ cysts [2-4]. Various supernumerary teeth are not seen in individuals with no other associated diseases/syndromes. It is usually related with cleft lip and palate, cleidocranial dysplasia and Gardner syndrome whereas there is no significant sex distribution in primary supernumerary teeth [4-7]. The excess teeth have an atypical crown which is vertical, horizontal /inverted position. They may grow and appear on the palate as extra teeth/they may grow in the nasal cavity. The teeth may be asymptomatic or cause a variety of signs and symptoms. Complications of nasal teeth are rhinitis caseosa with perforation, aspergillosisandoronasal fistula [2,3]. The diagnosis is done on the basis of clinical and radiographic findings. Clinically, an intranasal tooth is seen as a white mass in the nasal cavity surrounded. Radiographically, it seems radiopaque lesions with the similar attenuation as that of the oral teeth. With the bone window setting, the central radiolucency, which is correlated with the pulp cavity, may have a spot or slit, depending on the orientation of the teeth. Soft tissue found in clinical and pathologic examination, surrounds the radiopaque lesion and consistent with granulation tissue [4-7]. The differential diagnosis of nasal teeth is:

a. Radiopaque foreign body, b. Rhinolith,

c. Inflammatory lesions of syphilis, tuberculosis (or) fungal infection with calcification,

d. Benign tumours, as haemangioma, osteoma, calcified polyps, enchondroma,

e. Dermoid and malignant tumours, as chondrosarcoma and osteosarcoma.

Whereas, the Computed Tomography (CT) findings of toothequivalent attenuation and a centrally located cavity are highly discriminating features that are useful to approve the diagnosis. To remove nasal teeth, it is generally preferred to alleviate the symptoms and to avoid complications. When extra tooth is in the nasal cavity, the procedure followed is usually a minor operation. When a supernumerary tooth presents with a bony socket in the floor of the nose, its extraction may prove to be extremely challenging. CT is useful to assess the depth of site of eruption. The good time to remove the tooth is after the roots of the permanent teeth have completely formed, to prevent any injury while their development.

\section{Conclusion}

Supernumerary teeth are extra to normal complement in dentition. They may occur unilaterally or bilaterally, single or multiple. A variety of complications can occur ranging from crowding to cyst formation. Hence, early identification and treatment are essential for management.

\section{References}

1. Wurtele P, Dufour G (1994) Radiology case of the month: A tooth in the nose. J Otolaryngol 23(1): 67-68.

2. El Sayed Y (1995) Sinonasal teeth. J Otolaryngol 24(3): 180-183.

3. Liu JF (1995) Characteristics of premaxillary supernumerary teeth: A survey of 112 cases. ASDC J Dent Child 62(4): 262-265.

4. Scheiner MA, Sampson WJ (1997) Supernumerary teeth: A review of the literature and four case reports. Aust Dent J 42(3): 160-165.

5. Alexandrakis G, Hubbell RN, Aitken PA (2000) Nasolacrimal duct obstruction secondary to ectopic teeth. Ophthalmol 107(1): 189-192.

6. Chen A, Huang JK, Chang SJ, Sheu CY (2002) Nasal teeth: A report of three cases. Am J Neuroradiol 23(4): 671-673.

7. Gupta YK, Shah N (2001) Intranasal tooth as a complication of cleft lip and alveolus in four year old child: Case report and literature review. Int J Paediatr Dent 11(3): 221-224. 
This work is licensed under Creative Commons Attribution 4.0 License

To Submit Your Article Click Here: Submit Article

DOI: $10.32474 / S J 0.2020 .04 .000186$

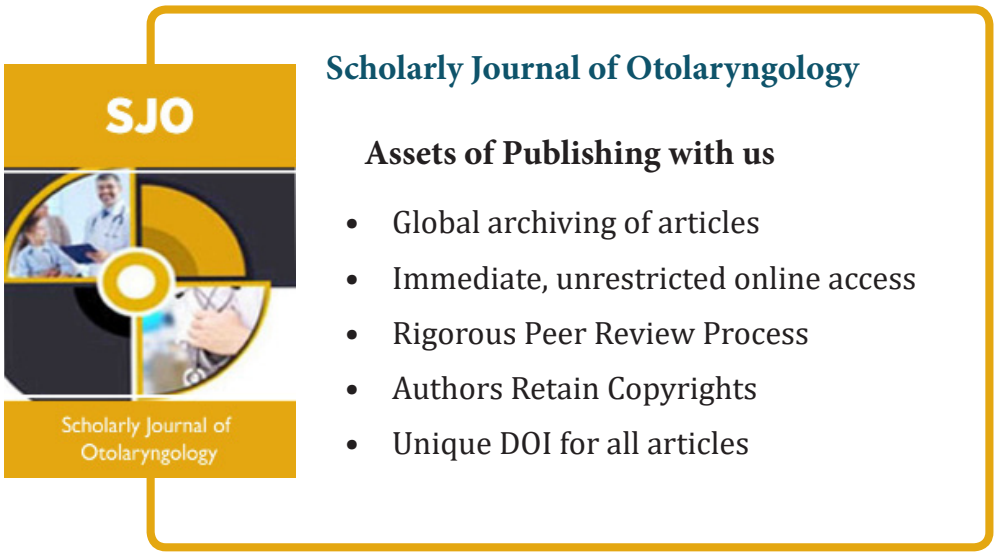

